\title{
Measurement of the effective weak mixing angle by jet-charge asymmetry in hadronic decays of the $Z$ boson
}

\section{L3 Collaboration}

M. Acciarri ${ }^{\text {aa }}$, O. Adriani ${ }^{\text {p }}$, M. Aguilar-Benitez ${ }^{\mathrm{z}}$, S. Ahlen ${ }^{\mathrm{k}}$, J. Alcaraz ${ }^{\mathrm{z}}$, G. Alemanni v, J. Allaby ${ }^{q}$, A. Aloisio ${ }^{\text {ac }}$, M.G. Alviggi ${ }^{\text {ac }}$, G. Ambrosi ${ }^{\text {s, }}$, H. Anderhub av , V.P. Andreev ak, T. Angelescu ${ }^{\text {m }}$, F. Anselmo ', A. Arefiev ${ }^{\text {ab }}$, T. Azemoon ${ }^{\text {c }}$, T. Aziz ${ }^{j}$, P. Bagnaia ${ }^{\text {aj }}$, L. Baksay ${ }^{\text {aq }}$, R.C. Ball ${ }^{\text {c }}$, S. Banerjee ${ }^{j}$, Sw. Banerjee ${ }^{j}, K_{\text {. Banicz }}{ }^{\text {as }}$, A. Barczyk ${ }^{\text {av,at }}$, R. Barillère ${ }^{q}$, L. Barone ${ }^{\text {aj, }}$, P. Bartalini v , A. Baschirotto ${ }^{\text {aa }}$, M. Basile ${ }^{\mathrm{i}}$, R. Battiston ${ }^{\mathrm{ag}}$, A. Bay ${ }^{\mathrm{v}}$, F. Becattini ${ }^{\mathrm{p}}$, U. Becker ${ }^{\circ}$, F. Behner av, J. Berdugo ${ }^{\mathrm{z}}$, P. Berges ${ }^{\circ}$, B. Bertucci ${ }^{\text {ag }}$, B.L. Betev ${ }^{\text {av }}$, S. Bhattacharya ${ }^{j}$, M. Biasini ${ }^{\text {ag }}$, A. Biland ${ }^{\text {av }}$, G.M. Bilei ${ }^{\text {ag }}$, J.J. Blaising ${ }^{d}$, S.C. Blyth ${ }^{\text {ah }}$, G.J. Bobbink ${ }^{\mathrm{b}}$, R. Bock ${ }^{\mathrm{a}}$, A. Böhm ${ }^{\mathrm{a}}$, L. Boldizsar ${ }^{\mathrm{n}}$, B. Borgia ${ }^{\text {q,aj, }}$

D. Bourilkov av , M. Bourquin s , D. Boutigny ${ }^{\text {d }}$, S. Braccini s, J.G. Branson ${ }^{\text {am }}$, V. Brigljevic ${ }^{\text {av }}$, I.C. Brock ${ }^{\text {ah }}$, A. Buffini ${ }^{\text {p }}$, A. Buijs ${ }^{\text {ar }}$, J.D. Burger ${ }^{\circ}$, W.J. Burger ${ }^{\text {ag }}$, J. Busenitz ${ }^{\text {aq }}$, X.D. Cai ${ }^{\circ}$, M. Campanelli ${ }^{\text {av }}$, M. Capell ${ }^{\circ}$, G. Cara Romeo ${ }^{\mathrm{i}}$, G. Carlino ${ }^{\text {ac }}$, A.M. Cartacci ${ }^{\text {p }}$, J. Casaus ${ }^{\text {z }}$, G. Castellini ${ }^{\text {p }}$, F. Cavallari aj, N. Cavallo ${ }^{\text {ac }}$, C. Cecchi ${ }^{\text {s }}$, M. Cerrada ${ }^{\text {z }}$, F. Cesaroni ${ }^{\text {w }}$, M. Chamizo ${ }^{\text {z }}$, Y.H. Chang ${ }^{a x}$, U.K. Chaturvedi ${ }^{r}$, M. Chemarin ${ }^{y}$, A. Chen ${ }^{a x}$, G. Chen ${ }^{g}$, G.M. Chen ${ }^{g}$, H.F. Chen ', H.S. Chen ${ }^{g}$, M. Chen ${ }^{\circ}$, G. Chiefari ${ }^{\text {ac }}$, C.Y. Chien ${ }^{\mathrm{e}}$, L. Cifarelli ${ }^{\text {al }}$, F. Cindolo ${ }^{\text {i }, ~ C . ~ C i v i n i n i ~}{ }^{\text {p }}$, I. Clare ${ }^{\circ}$, R. Clare ${ }^{\circ}$, G. Coignet ${ }^{d}$, A.P. Colijn ${ }^{\text {b }}$, N. Colino ${ }^{\text {z }}$, S. Costantini ${ }^{\text {h }}$, F. Cotorobai ${ }^{\mathrm{m}}$, B. de la Cruz ${ }^{\mathrm{z}}$, A. Csilling ${ }^{n}$, T.S. Dai ${ }^{\circ}$, R. D’Alessandro ${ }^{p}$, R. de Asmundis ${ }^{\text {ac }}$, A. Degré ${ }^{d}$, K. Deiters ${ }^{\text {at }}$, P. Denes ${ }^{\text {ai }}$, F. DeNotaristefani ${ }^{\text {aj, }}$ M. Diemoz ${ }^{\text {aj }}$, D. van Dierendonck ${ }^{\text {b }}$, F. Di Lodovico av , C. Dionisi ${ }^{\text {qaj }}$, M. Dittmar ${ }^{\text {av }}$, A. Dominguez ${ }^{\text {am }}$, A. Doria ${ }^{\text {ac }}$, M.T. Dova ${ }^{r, 1}$, E. Drago ${ }^{\text {ac }}$, D. Duchesneau ${ }^{\text {d }}$, P. Duinker ${ }^{b}$, I. Duran ${ }^{\text {an }}$, S. Easo ${ }^{\text {ag }}$, H. El Mamouni ${ }^{y}$, A. Engler ${ }^{\text {ah }}$, F.J. Eppling ${ }^{\circ}$, F.C. Erné ${ }^{b}$, P. Extermann ${ }^{\text {s, }}$ M. Fabre ${ }^{\text {at }}$, R. Faccini ${ }^{\text {aj }}$, M.A. Falagan ${ }^{\mathrm{z}}$, S. Falciano ${ }^{\text {aj }}$, A. Favara ${ }^{\mathrm{p}}$, J. Fay ${ }^{\mathrm{y}}$, O. Fedin ${ }^{\text {ak }}$, M. Felcini av ${ }^{\text {a }}$ T. Ferguson ${ }^{\text {ah }}$, F. Ferroni ${ }^{\text {aj }}$, H. Fesefeldt ${ }^{\text {a }}$, E. Fiandrini ${ }^{\text {ag }}$, J.H. Field ${ }^{\mathrm{s}}$, F. Filthaut ${ }^{\mathrm{q}}$, P.H. Fisher ${ }^{\circ}$, I. Fisk ${ }^{\text {am }}$, G. Forconi ${ }^{\circ}$, L. Fredj ${ }^{\mathrm{s}}$, K. Freudenreich ${ }^{\text {av }}$, C. Furetta ${ }^{\text {aa }}$, Yu. Galaktionov ${ }^{a b, o}$, S.N. Ganguli ${ }^{\mathrm{j}}$, 
P. Garcia-Abia ${ }^{\mathrm{f}}$, M. Gataullin af ${ }^{\text {a }}$, S.S. Gau ${ }^{1}$, S. Gentile ${ }^{\text {aj }}$, J. Gerald ${ }^{\text {e, }}$ N. Gheordanescu ${ }^{\mathrm{m}}$, S. Giagu ${ }^{\text {aj }}$, S. Goldfarb ${ }^{\mathrm{v}}$, J. Goldstein ${ }^{\mathrm{k}}$, Z.F. Gong ${ }^{\mathrm{t}}$, A. Gougas ${ }^{\text {e }}$, G. Gratta ${ }^{\text {af }}$, M.W. Gruenewald ${ }^{\text {h }}$, R. van Gulik ${ }^{\text {b }}$, V.K. Gupta ${ }^{\text {ai }}$, A. Gurtu ${ }^{j}$, L.J. Gutay as , D. Haas ${ }^{\text {f }}$, B. Hartmann ${ }^{a}$, A. Hasan ${ }^{\text {ad }}$, D. Hatzifotiadou ${ }^{\mathrm{i}}$, T. Hebbeker ${ }^{\text {h }}$, A. Hervé ${ }^{\mathrm{q}}$, P. Hidas ${ }^{\mathrm{n}}$, J. Hirschfelder ${ }^{\text {ah }}$, W.C. van Hoek ${ }^{\text {ae }}$, H. Hofer ${ }^{\text {av }}$, H. Hoorani ${ }^{\text {ah }}$, S.R. Hou ${ }^{\text {ax }}$, G. Hu ${ }^{\text {e }}$, I. Iashvili ${ }^{\text {au }}$, B.N. Jin ${ }^{\mathrm{g}}$, L.W. Jones ${ }^{c}$, P. de Jong ${ }^{q}$, I. Josa-Mutuberria ${ }^{z}$, A. Kasser ${ }^{\text {v }}$, R.A. Khan ${ }^{r}$, D. Kamrad ${ }^{\text {au }}$, J.S. Kapustinsky ${ }^{x}$, Y. Karyotakis ${ }^{\text {d }}$, M. Kaur ${ }^{\mathrm{r}, 2}$, M.N. Kienzle-Focacci s , D. Kim ${ }^{\text {aj }}$, D.H. Kim ${ }^{\text {ap }}$, J.K. Kim ${ }^{\text {ap }}$, S.C. Kim ${ }^{\text {ap }}$,

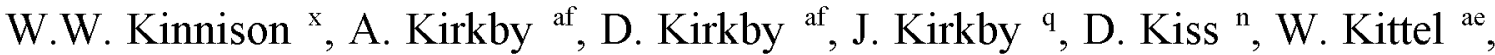
A. Klimentov ${ }^{\text {o,ab }}$, A.C. König ${ }^{\text {ae }}$, A. Kopp ${ }^{\text {au }}$, I. Korolko ${ }^{\text {ab }}$, V. Koutsenko ${ }^{\text {o,ab }}$, R.W. Kraemer ah, W. Krenz ${ }^{\text {a }}$, A. Kunin ${ }^{\text {o,ab }}$, P. Lacentre ${ }^{a u, 1,3}$,

P. Ladron de Guevara ${ }^{z}$, G. Landi ${ }^{p}$, C. Lapoint ${ }^{\circ}$, K. Lassila-Perini ${ }^{\text {av }}$, P. Laurikainen " ${ }^{\text {a }}$ A. Lavorato ${ }^{\text {al }}$, M. Lebeau ${ }^{\mathrm{q}}$, A. Lebedev ${ }^{\circ}$, P. Lebrun ${ }^{\mathrm{y}}$, P. Lecomte ${ }^{\text {av }}$, P. Lecoq ${ }^{q}$, P. Le Coultre ${ }^{\text {av }}$, H.J. Lee ${ }^{\text {h }}$, C. Leggett ${ }^{c}$, J.M. Le Goff ${ }^{q}$, R. Leiste ${ }^{\text {au }}$, E. Leonardi aj, P. Levtchenko ${ }^{\text {ak }}$, C. Li ${ }^{\mathrm{t}}$, C.H. Lin ${ }^{\text {ax }}$, W.T. Lin ${ }^{\text {ax }}$, F.L. Linde ${ }^{\mathrm{b}, \mathrm{q}}$, L. Lista ${ }^{\mathrm{ac}}$, Z.A. Liu ${ }^{\mathrm{g}}$, W. Lohmann ${ }^{\text {au }}$, E. Longo ${ }^{\text {aj }}$, W. Lu ${ }^{\text {af }}$, Y.S. Lu ${ }^{\text {g }}$, K. Lübelsmeyer ${ }^{\text {a }}$, C. Luci ${ }^{\text {q.aj }}$, D. Luckey ${ }^{\circ}$, L. Luminari aj, W. Lustermann av, W.G. Ma ${ }^{\mathrm{t}}$, M. Maity ${ }^{\mathrm{j}}$, G. Majumder ${ }^{\mathrm{j}}$, L. Malgeri ${ }^{\mathrm{q}}$, A. Malinin ${ }^{\text {ab }}$, C. Maña ${ }^{\text {z }}$, D. Mangeol ${ }^{\text {ae }}$, P. Marchesini ${ }^{\text {av }}$, G. Marian ${ }^{\text {aq, }}{ }^{4}$, A. Marin ${ }^{\text {k }}$, J.P. Martin y, F. Marzano aj, G.G.G. Massaro b , K. Mazumdar j, S. Mele ${ }^{\mathrm{q}}$, L. Merola $^{\text {ac }}$, M. Meschini ${ }^{\text {p }}$, W.J. Metzger ${ }^{\text {ae }}$, M. von der Mey ${ }^{a}$, Y. Mi v, D. Migani ${ }^{i}$, A. Mihul ${ }^{\mathrm{m}}$, A.J.W. van Mil ${ }^{\text {ae }}$, H. Milcent ${ }^{\mathrm{q}}$, G. Mirabelli ${ }^{\text {aj }}$, J. Mnich ${ }^{\mathrm{q}}$, P. Molnar ${ }^{\text {h }}$, B. Monteleoni ${ }^{\mathrm{p}}$, R. Moore ${ }^{\mathrm{c}}$, T. Moulik ${ }^{\mathrm{j}}$, R. Mount ${ }^{\text {af }}$, G.S. Muanza ${ }^{\mathrm{y}}$, F. Muheim ${ }^{\text {s }}$, A.J.M. Muijs ${ }^{\text {b }}$, S. Nahn ${ }^{\circ}$, M. Napolitano ${ }^{\text {ac }}$, F. Nessi-Tedaldi ${ }^{\text {av }}$, H. Newman ${ }^{\text {af }}$, T. Niessen ${ }^{\text {a }}$, A. Nippe ${ }^{\mathrm{v}}$, A. Nisati ${ }^{\text {aj }}$, H. Nowak ${ }^{\text {au }}$, Y.D. Oh ${ }^{\text {ap }}$, G. Organtini aj, R. Ostonen ", C. Palomares ${ }^{z}$, D. Pandoulas a, S. Paoletti aj,q, P. Paolucci ${ }^{\text {ac }}$, H.K. Park ${ }^{\text {ah }}$, I.H. Park ${ }^{\text {ap }}$, G. Pascale ${ }^{\text {aj }}$, G. Passaleva ${ }^{\text {q }}$, S. Patricelli ${ }^{\text {ac }}$, T. Paul ${ }^{~}$, M. Pauluzzi ${ }^{\text {ag }}$, C. Paus ${ }^{\mathrm{q}}$, F. Pauss ${ }^{\text {av }}$, D. Peach ${ }^{\mathrm{q}}$, M. Pedace aj, Y.J. Pei a S. Pensotti ${ }^{\text {aa }}$, D. Perret-Gallix ${ }^{\mathrm{d}}, \mathrm{B}$. Petersen ${ }^{\text {ae }}, \mathrm{S}$. Petrak ${ }^{\mathrm{h}}$, A. Pevsner ${ }^{\mathrm{e}}$, D. Piccolo ${ }^{\text {ac }}$, M. Pieri ${ }^{\text {p }}$, P.A. Piroué ${ }^{\text {ai }}$, E. Pistolesi ${ }^{\text {aa }}$, V. Plyaskin ${ }^{\text {ab }}$, M. Pohl ${ }^{\text {av }}$, V. Pojidaev ${ }^{a b, p}$, H. Postema ${ }^{o}$, J. Pothier ${ }^{\mathrm{q}}$, N. Produit s, D. Prokofiev ak, J. Quartieri al, G. Rahal-Callot av, N. Raja ${ }^{\text {j, P.G. Rancoita }}{ }^{\text {aa }}$, M. Rattaggi ${ }^{\text {aa }}$, G. Raven ${ }^{\text {am }}$, P. Razis ${ }^{\text {ad }}$, D. Ren ${ }^{\text {av }}$, M. Rescigno ${ }^{\text {aj, }}$ S. Reucroft ${ }^{1}$, T. van Rhee ${ }^{\text {ar }}$, S. Riemann ${ }^{\text {au }}$, K. Riles ${ }^{\text {c }}$, O. Rind ${ }^{\text {c }}$, A. Robohm ${ }^{\text {av }}$, J. Rodin ${ }^{\text {aq }}$, B.P. Roe ${ }^{\text {, }}$,

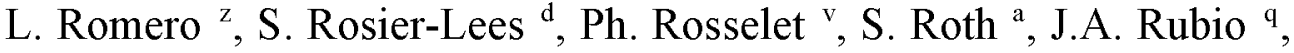
D. Ruschmeier ${ }^{\text {h }}$, H. Rykaczewski av, S. Sakar ${ }^{\text {aj }}$, J. Salicio ${ }^{\text {q }}$, E. Sanchez ${ }^{z}$, M.P. Sanders ae, M.E. Sarakinos ", G. Sauvage ${ }^{\text {d }, ~ C . ~ S c h a ̈ f e r ~ a, ~ V . ~ S c h e g e l s k y ~}{ }^{\text {ak }}$, 
S. Schmidt-Kaerst a , D. Schmitz a , M. Schneegans ${ }^{\text {d }}$, N. Scholz av, H. Schopper ${ }^{\text {aw }}$, D.J. Schotanus ae, J. Schwenke a , G. Schwering a , C. Sciacca ${ }^{\text {ac }}$, D. Sciarrino ${ }^{\text {s, }}$, L. Servoli ag, S. Shevchenko af, N. Shivarov ${ }^{\text {ao, }}$, V. Shoutko ab, J. Shukla ${ }^{\mathrm{x}}$, E. Shumilov ${ }^{\mathrm{ab}}$, A. Shvorob af ${ }^{\text {, T. Siedenburg }}{ }^{\text {a }}$, D. Son ${ }^{\text {ap }}$, V. Soulimov ${ }^{\text {ac }}$, B. Smith ${ }^{\circ}$, P. Spillantini ${ }^{\mathrm{p}}$, M. Steuer ${ }^{\circ}$, D.P. Stickland ${ }^{\text {ai }}$, H. Stone ${ }^{\text {ai }}$, B. Stoyanov ${ }^{\text {ao }}$, A. Straessner ${ }^{\text {a }}$, K. Sudhakar ${ }^{\text {j, G. Sultanov }}{ }^{\mathrm{r}}$, L.Z. Sun ${ }^{\mathrm{t}}$, G.F. Susinno ${ }^{\text {s, H. Suter }}{ }^{\text {av }}$, J.D. Swain ${ }^{\mathrm{r}}$, X.W. Tang ${ }^{\mathrm{g}}$, L. Tauscher ${ }^{\mathrm{f}}$, L. Taylor ${ }^{1}$, C. Timmermans ${ }^{\text {ae }}$, Samuel C.C. Ting ${ }^{\circ}$, S.M. Ting ${ }^{\circ}$, S.C. Tonwar ${ }^{j}$, J. Tóth ${ }^{n}$, C. Tully ${ }^{\text {ai }}$, K.L. Tung ${ }^{\mathrm{g}}$, Y. Uchida ${ }^{\circ}$, J. Ulbricht ${ }^{\text {av }}$, E. Valente ${ }^{\text {aj }}$, G. Vesztergombi ${ }^{\text {n }}$, I. Vetlitsky ${ }^{\text {ab }}$, G. Viertel ${ }^{\text {av }}$, S. Villa ${ }^{\text {l }}$, M. Vivargent ${ }^{\text {d }}$, S. Vlachos ${ }^{f}$, H. Vogel ${ }^{\text {ah }}$, H. Vogt ${ }^{\text {au }}$, I. Vorobiev ${ }^{\text {q,ab }}$, A.A. Vorobyov ${ }^{\text {ak }}$, A. Vorvolakos ${ }^{\text {ad }}$, M. Wadhwa ${ }^{f}$, W. Wallraff a , J.C. Wang ${ }^{\circ}$, X.L. Wang ${ }^{\mathrm{t}}$, Z.M. Wang ${ }^{\mathrm{t}}$, A. Weber ${ }^{\mathrm{a}}$, S.X. Wu ${ }^{\circ}$, S. Wynhoff ${ }^{\text {a }}$, J. Xu ${ }^{\text {k }}$, Z.Z. Xu ${ }^{\text {t, B.Z. Yang }}{ }^{\text {t }, ~ C . G . ~ Y a n g ~}{ }^{\mathrm{g}}$, H.J. Yang ${ }^{\mathrm{g}}$, M. Yang ${ }^{\mathrm{g}}$, J.B. Ye ${ }^{\mathrm{t}}$, S.C. Yeh ${ }^{\text {ay }}$, J.M. You ${ }^{\text {ah }}$, An. Zalite ${ }^{\text {ak }}$, Yu. Zalite ${ }^{\text {ak }}$, P. Zemp ${ }^{\text {av }}$, Y. Zeng ${ }^{\text {a }}$, Z.P. Zhang ${ }^{\text {t, B. Zhou }}{ }^{\text {k, }}$ Y. Zhou ${ }^{\text {c }}$, G.Y. Zhu ${ }^{\text {g }}$, R.Y. Zhu ${ }^{\text {af }}$, A. Zichichi i, ${ }^{\text {i,r }}$, F. Ziegler ${ }^{\text {au }}$, G. Zilizi aq,4

a I. Physikalisches Institut, RWTH, D-52056 Aachen, Germany, and III. Physikalisches Institut, RWTH, D-52056 Aachen, Germany ${ }^{5}$

${ }^{\mathrm{b}}$ National Institute for High Energy Physics, NIKHEF, and University of Amsterdam, NL-1009 DB Amsterdam, Netherlands ${ }^{c}$ University of Michigan, Ann Arbor, MI 48109, USA

${ }^{d}$ Laboratoire d'Annecy-le-Vieux de Physique des Particules, LAPP, IN2P3-CNRS, BP 110, F-74941 Annecy-le-Vieux CEDEX, France

' Johns Hopkins University, Baltimore, MD 21218, USA

${ }^{\mathrm{f}}$ Institute of Physics, University of Basel, $\mathrm{CH}-4056$ Basel, Switzerland

${ }^{\mathrm{g}}$ Institute of High Energy Physics, IHEP, 100039 Beijing, China ${ }^{6}$

${ }^{\mathrm{h}}$ Humboldt University, D-10099 Berlin, Germany ${ }^{5}$

${ }^{\mathrm{i}}$ University of Bologna and INFN-Sezione di Bologna, I-40126 Bologna, Italy

${ }^{\mathrm{j}}$ Tata Institute of Fundamental Research, Bombay 400005 , India

${ }^{\mathrm{k}}$ Boston University, Boston, MA 02215, USA

${ }^{1}$ Northeastern University, Boston, MA 02115, USA

$\mathrm{m}$ Institute of Atomic Physics and University of Bucharest, R-76900 Bucharest, Romania

${ }^{\mathrm{n}}$ Central Research Institute for Physics of the Hungarian Academy of Sciences, $\mathrm{H}-1525$ Budapest 114, Hungary ${ }^{7}$

'Massachusetts Institute of Technology, Cambridge, MA 02139, USA

${ }^{\mathrm{P}}$ INFN Sezione di Firenze and University of Florence, I-50125 Florence, Italy

${ }^{\mathrm{q}}$ European Laboratory for Particle Physics, CERN, CH-1211 Geneva 23, Switzerland

${ }^{\mathrm{r}}$ World Laboratory, FBLJA Project, CH-1211 Geneva 23, Switzerland

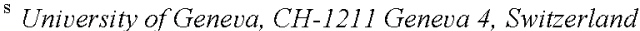

${ }^{t}$ Chinese University of Science and Technology, USTC, Hefei, Anhui 230 029, China ${ }^{6}$

"SEFT, Research Institute for High Energy Physics, P.O. Box 9, SF-00014 Helsinki, Finland

"University of Lausanne, $\mathrm{CH}-1015$ Lausanne, Switzerland

${ }^{w}$ INFN-Sezione di Lecce and Universitá Degli Studi di Lecce, I-73100 Lecce, Italy

${ }^{x}$ Los Alamos National Laboratory, Los Alamos, NM 87544, USA

y Institut de Physique Nucléaire de Lyon, IN2P3-CNRS, Université Claude Bernard, F-69622 Villeurbanne, France

${ }^{\mathrm{z}}$ Centro de Investigaciones Energeticas, Medioambientales y Tecnologicas, CIEMAT, E-28040Madrid, Spain ${ }^{8}$

${ }^{\text {aa } I N F N-S e z i o n e ~ d i ~ M i l a n o, ~ I-20133 ~ M i l a n, ~ I t a l y ~}$

ab Institute of Theoretical and Experimental Physics, ITEP, Moscow, Russia

ac INFN-Sezione di Napoli and University of Naples, I-80125 Naples, Italy

ad Department of Natural Sciences, University of Cyprus, Nicosia, Cyprus

${ }^{\text {ae }}$ University of Nijmegen and NIKHEF, NL-6525 ED Nijmegen, Netherlands

af California Institute of Technology, Pasadena, CA 91125, USA

ag INFN-Sezione di Perugia and Universitá Degli Studi di Perugia, I-06100 Perugia, Italy 
ah Carnegie Mellon University, Pittsburgh, PA 15213, USA

ai Princeton University, Princeton, NJ 08544, USA

aj INFN-Sezione di Roma and University of Rome, "La Sapienza", I-00185 Rome, Italy

${ }^{\mathrm{ak}}$ Nuclear Physics Institute, St. Petersburg, Russia

al University and INFN, Salerno, I-84100 Salerno, Italy

am University of California, San Diego, CA 92093, USA

an Dept. de Fisica de Particulas Elementales, Univ. de Santiago, E-15706 Santiago de Compostela, Spain

ao Bulgarian Academy of Sciences, Central Lab. of Mechatronics and Instrumentation, BU-1113 Sofia, Bulgaria

ap Center for High Energy Physics, Adv. Inst. of Sciences and Technology, 305-701 Taejon, South Korea

aq University of Alabama. Tuscaloosa, AL 35486, USA

ar Utrecht University and NIKHEF, NL-3584 CB Utrecht, Netherlands

as Purdue University, West Lafayette, IN 47907, USA

${ }^{\text {at }}$ Paul Scherrer Institut, PSI, CH-5232 Villigen, Switzerland

au DESY-Institut fur Hochenergiephysik, D-15738 Zeuthen, Germany

av Eidgenössische Technische Hochschule, ETH Zürich, CH-8093 Zürich, Switzerland

${ }^{\text {aw }}$ University of Hamburg, D-22761 Hamburg, Germany

ax National Central University, Chung-Li, Taiwan, China

ay Department of Physics, National Tsing Hua University, Taiwan, China

Received 12 August 1998

Editor: K. Winter

\begin{abstract}
The coupling of the $Z$ boson to quarks is studied in a sample of about 3.5 million hadronic $Z$ decays collected by the L 3 experiment at LEP from 1991 to 1995. The forward-backward quark charge asymmetry is measured by means of a jet charge technique. From the measured asymmetries, the effective weak mixing angle is determined to be $\sin ^{2} \bar{\theta}_{\mathrm{W}}=0.2327 \pm$ 0.0012 (stat. $) \pm 0.0013$ (syst.). (C) 1998 Published by Elsevier Science B.V. All rights reserved.
\end{abstract}

\section{Introduction}

The Standard Model of electroweak interactions [1] predicts a mixing between the two mediators of neutral currents. Consequently, the coupling constants of the $Z$ boson acquire a dependence on the

\footnotetext{
${ }^{1}$ Also supported by CONICET and Universidad Nacional de La Plata, CC 67, 1900 La Plata, Argentina.

${ }^{2}$ Also supported by Panjab University, Chandigarh-160014, India.

${ }^{3}$ Supported by Deutscher Akademischer Austauschdienst.

${ }^{4}$ Also supported by the Hungarian OTKA fund under contract numbers T22238 and T026178.

${ }^{5}$ Supported by the German Bundesministerium für Bildung, Wissenschaft, Forschung und Technologie.

${ }^{6}$ Supported by the National Natural Science Foundation of China.

${ }^{7}$ Supported by the Hungarian OTKA fund under contract numbers T019181, F023259 and T024011.

${ }^{8}$ Supported also by the Comisión Interministerial de Ciencia y Technología.
}

weak mixing angle $\sin ^{2} \theta_{\mathrm{W}}$. Measuring the forwardbackward asymmetries in $Z$ boson decays therefore allows to determine $\sin ^{2} \theta_{\mathrm{W}}$.

In the process $\mathrm{e}^{+} \mathrm{e}^{-} \rightarrow q \bar{q}$ the distribution of the quark production angle, $\theta$, relative to the $\mathrm{e}^{-}$beam direction, can be parametrised by

$$
\frac{d \sigma}{d \cos \theta} \sim 1+\cos ^{2} \theta+\frac{8}{3} A_{\mathrm{fb}}^{q} \cos \theta .
$$

At lowest order, for a centre-of-mass energy, $\sqrt{s}$, equal to $m_{Z}$ the forward-backward asymmetry becomes

$A_{\mathrm{fb}}^{q}\left(m_{\mathrm{Z}}\right)=\frac{3}{4} A_{\mathrm{e}} A_{q}, \quad A_{f}=\frac{2 v_{f} a_{f}}{v_{f}^{2}+a_{f}^{2}}$.

Higher-order weak corrections are taken into account by replacing the couplings $v_{f}$ and $a_{f}$ by effective couplings $\bar{v}_{f}$ and $\bar{a}_{f}$ which are related to the effective weak mixing angle, $\sin ^{2} \bar{\theta}_{\mathrm{W}}[2]$, by

$\bar{v}_{f} / \bar{a}_{f}=1-4\left|Q_{f}\right| \sin ^{2} \bar{\theta}_{\mathrm{W}}$. 
In contrast to the leptonic final states, the analysis of hadronic final states is complicated by the fragmentation process, which obscures a charge measurement of the original quarks. In addition hadronic final states are a mixture of up- and down-type quark pairs, reducing the sensitivity of the forward-backward asymmetry measurement to the value of $\sin ^{2} \bar{\theta}_{\mathrm{W}}$. Previous measurements of the quark charge asymmetry have been performed at LEP [3-5].

This paper describes the measurement of the quark charge asymmetry in a sample of about 3.5 million hadronic $Z$ decays. The data were collected between 1991 and 1995 by the L3 experiment at centre-ofmass energies around the $Z$ peak, corresponding to an integrated luminosity of $135 \mathrm{pb}^{-1}$. A jet-charge technique [6] is used to identify the quark charge signs.

The analysis is performed as follows: a flavouraveraged probability to correctly identify the charge sign of the quarks is determined from the data. This is used to extract raw asymmetries. These raw asymmetries are fitted, in the Standard Model framework, to determine the value of $\sin ^{2} \bar{\theta}_{\mathrm{W}}$. For the data taken in 1994 and 1995 the identification of heavy flavours is used to increase the sensitivity to $\sin ^{2} \vec{\theta}_{\mathrm{W}}$ and to measure the $\mathrm{b} \overline{\mathrm{b}}$ forward-backward asymmetry, $A_{\mathrm{fb}}^{\mathrm{b}}$, in a more model-independent way.

\section{Event selection}

The data is collected with the L3 detector [7-11] and is analysed separately for each year and centreof-mass energy point. Hadronic events are selected by cuts similar to those used in Ref. [12]:

- visible energy: $0.5 \leq E_{\mathrm{vis}} / \sqrt{s} \leq 1.5$

- transverse energy imbalance: $E_{\mathrm{tran}} / E_{\mathrm{vis}} \leq 0.5$

- longitudinal energy imbalance: $E_{\text {long }} / E_{\mathrm{vis}} \leq 0.5$

- number of calorimetric energy deposits: $N_{\mathrm{cl}}>13$

- polar angle of the thrust axis: $\left|\cos \theta_{t}\right|<0.85$.

The events are further divided into two hemispheres separated by the plane perpendicular to the thrust axis. For the analysis only events are considered which have at least two tracks reconstructed in one hemisphere and at least one track in the other one. Tracks in the central tracker are required to have a measured transverse momentum between 1 and 50 $\mathrm{GeV}$ and to originate from the interaction point.
Table 1

Centre-of-mass energy, $\sqrt{s}$, integrated luminosity, $\mathscr{L}$, and number of selected events, $N_{\text {sel }}$, for the 15 data periods

\begin{tabular}{llcc}
\hline Year & $\sqrt{s}(\mathrm{GeV})$ & $\mathscr{L}\left(\mathrm{nb}^{-1}\right)$ & $N_{\text {sel }}\left(10^{3}\right)$ \\
\hline 1991 & 88.48 & 739.3 & 3.1 \\
& 89.47 & 766.1 & 6.1 \\
& 90.23 & 748.4 & 10.6 \\
& 91.24 & 7258 & 172.5 \\
& 91.97 & 648.1 & 12.5 \\
& 92.97 & 731.0 & 8.2 \\
& 93.72 & 750.1 & 5.8 \\
1992 & 91.29 & 20473 & 480.1 \\
1993 & 89.44 & 8538 & 67.6 \\
& 91.24 & 15172 & 363.8 \\
& 93.03 & 8806 & 96.8 \\
1994 & 91.22 & 44550 & 967.8 \\
1995 & 89.45 & 7391 & 53.4 \\
& 91.31 & 10101 & 234.9 \\
& 92.99 & 8275 & 89.4 \\
\hline
\end{tabular}

In Table 1 the centre-of-mass energy, the collected luminosity and the number of events used in the following analysis are listed for each period.

The Monte Carlo samples of hadronic $Z$ decays used for fragmentation studies are generated using the PYTHIA/JETSET program [13]. The samples of $\mathrm{e}^{+} \mathrm{e}^{-} \rightarrow \tau^{+} \tau^{-}(\gamma)$ and $\mathrm{e}^{+} \mathrm{e}^{-} \rightarrow \mathrm{e}^{+} \mathrm{e}^{-} q \bar{q}$ events used in the evaluation of the background contamination are generated with the KORALZ [14] and the DIAG36 [15] programs, respectively. These backgrounds contributions are small: $0.3 \%$ and $0.2 \%$. The response of the $\mathrm{L} 3$ detector is modelled with the GEANT [16] detector simulation program which includes the effects of energy loss, multiple scattering and showering in the detector materials and in the beampipe.

\section{Measurement techniques}

\subsection{Jet-charge measurement}

The thrust axis is used to estimate the direction of flight of the quark-pair produced in the $Z$ decay. For each hemisphere, defined by the thrust axis, the jet charge is given by

$Q_{h}=\frac{\sum_{i} q_{i} w_{i}}{N_{\text {trk }}}, \quad h=\mathrm{F}, \mathrm{B}$, 


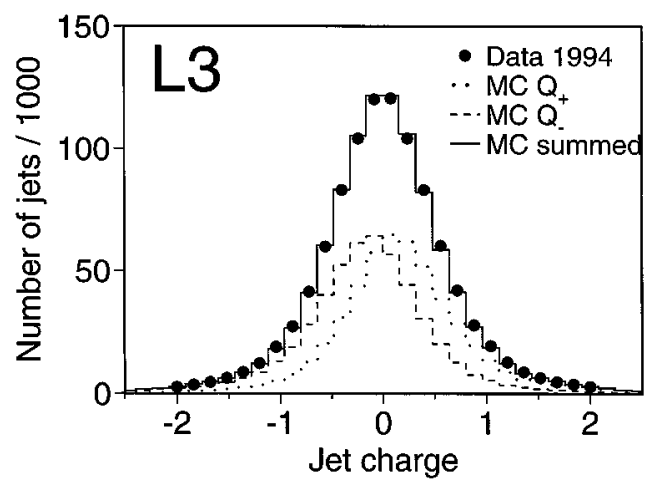

Fig. 1. $Q_{+}$and $Q_{-}$distributions obtained from Monte Carlo. Also their sum is compared to the sum of the $Q_{\mathrm{F}}$ and $Q_{\mathrm{B}}$ distributions for 1994 data.

where forward (F) and backward (B) are defined with respect to the direction of the $\mathrm{e}^{-}$beam. The index $i$ runs over all tracks per hemisphere, $N_{\text {trk }}$. The charge and weight per track are $q_{i}$ and $w_{i}$, respectively. The weight is determined from the track momentum component along the thrust direction, $p_{i}^{\|}$, and the track charge confusion, $C$, as

$w_{i}=\left|p_{i}^{\|}\right|^{\kappa} \times\left(1-C\left(\phi^{i}\right)\right)$.

The track charge confusion depends on the azimuthal angle, $\phi^{\prime}$, of the track, and is estimated using leptonic final state data. An event is called forward if $Q_{\mathrm{F}}$ is larger than $Q_{\mathrm{B}}$. The parameter $\kappa$, which is a free parameter of the method [6], is set to 0.4 to maximise the probability, $P_{\mathrm{S}}$, for a correct assignment of the event being forward or backward.

The jet charge defined in Eq. (4) is used to discriminate between jets originating from positively and negatively charged quarks. Denoting the jet charge of positively and negatively charged quarks by $Q_{+}$and $Q_{-}$, respectively, it is assumed that the distributions of $Q_{+}$and $Q_{-}$are Gaussians and have mean values differing by a charge separation $\delta_{Q}$, variances $\sigma_{Q}$ and a correlation $\rho$. Then the probability $P_{\mathrm{S}}$ is given by

$P_{\mathrm{S}}=\frac{1}{\sqrt{2 \pi}} \int_{x_{0}}^{\infty} \mathrm{e}^{-\frac{1}{2} x^{2}} d x, \quad x_{0}=\frac{-\delta_{Q}}{\sigma_{Q} \sqrt{2(1-\rho)}}$.

The quantities $\delta_{O}$ and $\sigma_{O}$ are extracted from the data in the following way: A distribution $Q$ is con- structed, equal to the sum of the $Q_{+}$and $Q_{-}$ distributions. For the variance of this distribution, $V(Q)$, and the correlation coefficient, $\rho$, the following relations hold:

$V(Q)=\sigma_{Q}^{2}+\frac{1}{4} \delta_{Q}^{2}$,

$\rho \sigma_{Q}^{2}=\left\langle Q_{+} Q_{-}\right\rangle-\left\langle Q_{+}\right\rangle\left\langle Q_{-}\right\rangle$.

The brackets denote the mean value of the distribution. From these relations the charge separation is determined to be

$\delta_{Q}^{2}=\frac{4\left(\rho V(Q)+\langle Q\rangle^{2}-\left\langle Q_{+} Q_{-}\right\rangle\right)}{1+\rho}$.

Because the $Q$ distribution also represents the sum of the $Q_{\mathrm{F}}$ and $Q_{\mathrm{B}}$ distributions, and as $\left\langle Q_{\mathrm{F}} Q_{\mathrm{B}}\right\rangle=$ $\left\langle Q_{+} Q_{-}\right\rangle, \delta_{Q}$ and $\sigma_{Q}$ can be calculated using these quantities, measured on data, and the correlation coefficient $\rho$, which is the only quantity that is determined from Monte Carlo events. In Fig. 1 the Monte Carlo distributions of the jet charges of the positive and negative quark hemispheres are shown; also the $Q$ distribution is compared to the data.

Applying the same method to a sample of fully simulated Monte Carlo events, the estimated $P_{\mathrm{S}}$ values are shown to reproduce well the fractions of a correct charge assignment, $P_{\text {gen }}$, as determined using generator-level information. This is shown in Fig. 2 which includes also the distribution obtained for the data taken in 1993. A good agreement between the

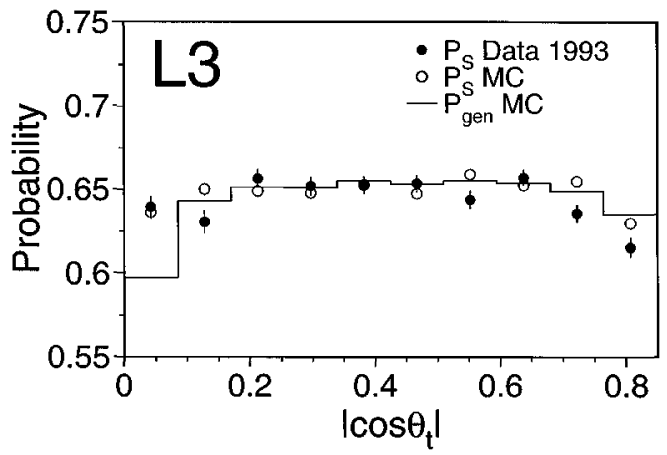

Fig. 2. Probability of a correct charge assignment versus $\left|\cos \theta_{t}\right|$. The solid and open points represent the calculated $P_{\mathrm{S}}$ values for 1993 data and Monte Carlo, respectively; the histogram represents the fraction of a correct charge assignment, $P_{\mathrm{gcn}}$, as evaluated using the Monte Carlo generator information. 
Monte Carlo values is obtained, except for the first bin. The discrepancy observed here is an artefact of the comparison, caused by the migration of events between forward and backward directions due to the thrust axis angular resolution. This affects the evaluation made using generator-level information, but not the procedure used to extract $\sin ^{2} \bar{\theta}_{w}$ where the resolution is accounted for in the fit to determine the raw asymmetries. Typical average values of $P_{\mathrm{S}}$ are $64.0 \%$ in 1991 and $66.5 \%$ in 1994 .

These low values of $P_{\mathrm{S}}$ are mainly due to fragmentation effects. The charge identification using a jet charge technique assumes that the highest-energy particles produced in the hadronisation carry the information of the sign of the charge of the original quark. This charge information is diluted for several reasons:

- Primary mesons ${ }^{9}$ may be neutral, which is more likely for down-type quarks than for up-type ones. This is due to the possibility to create SS pairs in the fragmentation process, in addition to u $\bar{u}$ and $\mathrm{d} \overline{\mathrm{d}}$.

- Up-type quarks are more likely to produce charged than neutral baryons; the converse is true for down-type quarks. This reduces the correlation between jet charges and quark charges for downtype quarks relative to up-type quarks.

- The decay of mesons, in particular in case of heavy flavour mesons, distributes the momentum among several particles.

The differences in $P_{\mathrm{S}}$ among the flavours caused by these effects are estimated using Monte Carlo event samples. The relevant parameters of the JETSET hadronisation model on which these estimates depend are given in Section 4.2.

\subsection{Flavour tagging method}

For the data taking periods where the silicon microvertex detector was operational (1994 and 1995) an impact parameter flavour tagging method, described in detail in Ref. [17], is used to distinguish

\footnotetext{
${ }^{9}$ The primary meson of a jet is a bound state of the original quark/antiquark and the antiquark/quark of the first quark pair produced in the hadronisation.
}

decays of the $Z$ into heavy quarks. The distance of closest approach in the transverse plane to the primary vertex of the event is calculated for each track. The corresponding error is evaluated taking into account both the uncertainties on the vertex and those on the track parameters. A sign is given to the impact parameter, according to the position of the intersection between the track and the jet direction with respect to the primary vertex. The tracks with negative impact parameters are likely to be fragmentation tracks. From the impact parameters and their errors the probability of the individual tracks to originate from the interaction point is estimated. The probability, $P_{\mathrm{E}}$, that there is no secondary vertex is evaluated by combining all the tracks of the event. The discriminator, $D_{\mathrm{tag}}$, used to tag quark flavours is given by $D_{\text {tag }}=-\log _{10}\left(P_{\mathrm{E}}\right)$. The $D_{\text {tag }}$ distribution is shown for 1995 data in Fig. 3(a).
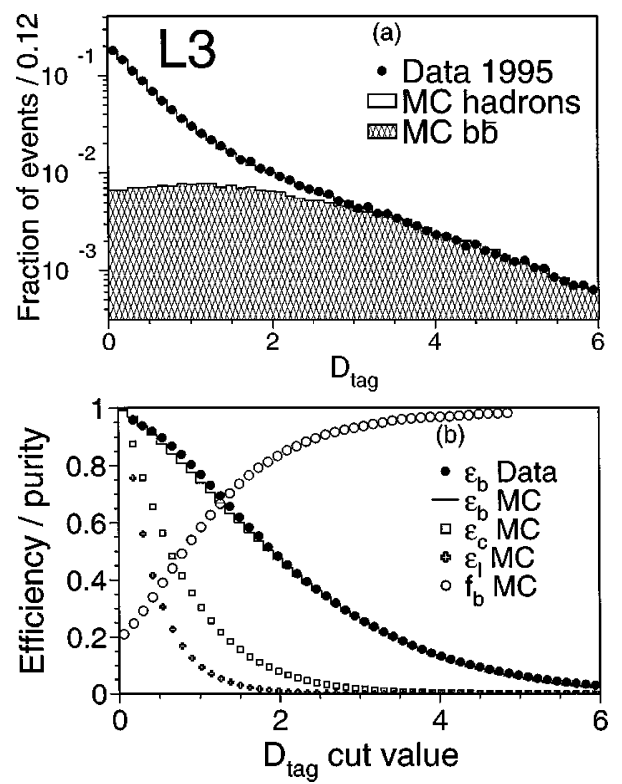

Fig. 3. Performance of the flavour tagging algorithm: (a) $D_{\text {tag }}$ distribution in data and $\mathrm{MC}$. The contribution of the events with $b$ quarks is indicated by the hatched histogram. (b) efficiencies $(\varepsilon)$ for the different flavours as a function of the $D_{\text {tag }}$ cut value based on Monte Carlo calculations. The index $l$ denotes the light flavours. For b quarks also the efficiency obtained from data is given, as well as the purity $\left(f_{\mathrm{b}}\right)$ obtained from Monte Carlo. 


\section{Measurement of asymmetries}

\subsection{Extraction of raw asymmetries}

The data taken in each year and at each centreof-mass energy point are considered separately. In addition, the data taken on the $Z$ peak in 1994 and 1995 are split into 8 samples, corresponding to different values of $D_{\text {tag }}$. Bins are chosen in order to contain similar numbers of events per bin.

In each of these samples an unbinned maximum likelihood fit is performed to the distribution of

$x=\operatorname{sign}\left(Q_{\mathrm{F}}-Q_{\mathrm{B}}\right)\left|\cos \theta_{\mathrm{t}}\right|$.

The variable $x$ represents with a probability $P_{\mathrm{S}}$ the direction of flight of the positive quark originating from the $Z$ decay with respect to the $\mathrm{e}^{-}$beam direction. The likelihood function is then calculated from the expected angular distribution for a given $A_{\mathrm{fb}}^{\mathrm{raw}}$ :

$$
\begin{aligned}
\mathscr{L}\left(A_{\mathrm{fb}}^{\mathrm{raw}}\right)= & \prod_{i=1}^{N_{\mathrm{evt}}} f\left(x_{i}, A_{\mathrm{fb}}^{\mathrm{raw}}\right), \\
f\left(x, A_{\mathrm{fb}}^{\mathrm{raw}}\right)= & 1+a x^{2} \\
& +\frac{6+2 a}{3} A_{\mathrm{fb}}^{\mathrm{raw}}\left(2 P_{\mathrm{S}}(x)-1\right) x .
\end{aligned}
$$

The coefficient $a=0.952 \pm 0.009$ is introduced to account for the deformation of the polar angular distribution due to QCD corrections [18], and is flavour-independent to a good approximation. The thrust axis angular resolution is accounted for by convoluting expression (Eq. (10)) with a Gaussian.
The raw asymmetries, $A_{\mathrm{fb}}^{\text {raw }}$, which result from the fit, correspond to a linear combination of the forward-backward asymmetries $A_{\mathrm{fb}}^{q}$ introduced in Eq. (1):

$A_{\mathrm{fb}}^{\mathrm{raw}}=\sum_{q} \frac{Q_{q}}{\left|Q_{q}\right|} f_{q} \xi_{q}\left(1-C_{q}\right) A_{\mathrm{fb}}^{q}$,

$f_{q}=\varepsilon_{q} R_{q} / \sum_{q^{\prime}} \varepsilon_{q^{\prime}} R_{q^{\prime}}$,

where the $C_{q}$ are flavour-dependent QCD corrections [18], the $\varepsilon_{q}$ are the selection efficiencies of the various flavours in the sample, and the $R_{q}=$ $\sigma\left(\mathrm{e}^{+} \mathrm{e}^{-} \rightarrow q \bar{q}\right) / \Sigma_{q^{\prime}} \sigma\left(\mathrm{e}^{+} \mathrm{e}^{-} \rightarrow q^{\prime} q^{\prime}\right)$ are the relative cross sections. The flavour biases, $\xi_{q}$, take into account the difference in the probability for correct charge assignment between the flavours and are given by

$\xi_{q}=\frac{2 P_{q}-1}{2 P_{\mathrm{S}}-1}$,

where the $P_{q}$ are scaled to yield the same average $P_{\mathrm{S}}$ as obtained from the data. A Monte Carlo study showed that the polar angular dependence of the $\xi_{q}$ can be neglected.

The raw asymmetries measured in 1994 and 1995 for the 8 bins of the tagging variable $D_{\text {tag }}$ are listed in Table 2. Also given are the fractions of uds, $\mathrm{c}$, and b events for each $D_{\text {tag }}$ bin.

The flavour biases depend on the performance of the tracking and are therefore determined for each year separately. For example, values found for the five quark flavours in 1994 are: $\xi_{\mathrm{u}}=1.41, \xi_{\mathrm{d}}=$ $0.88, \xi_{\mathrm{s}}=1.07, \xi_{\mathrm{c}}=0.85$ and $\xi_{\mathrm{b}}=0.87$.

\begin{tabular}{|c|c|c|c|c|c|}
\hline$D_{\text {tag }}$ range & \multicolumn{2}{|l|}{$A_{\mathrm{fb}}^{\mathrm{raw}}(\%)$} & $\varepsilon_{\mathrm{usd}}$ & $\varepsilon_{\mathrm{c}}$ & $\varepsilon_{\mathrm{b}}$ \\
\hline$<0.06$ & $-2.70 \pm 0.79$ & $-1.33 \pm 1.53$ & 0.245 & 0.134 & 0.0328 \\
\hline $0.18-0.30$ & $-3.48 \pm 0.96$ & $-1.32 \pm 1.95$ & 0.147 & 0.114 & 0.0379 \\
\hline $0.30-0.54$ & $-1.64 \pm 0.80$ & $0.62 \pm 1.64$ & 0.183 & 0.180 & 0.0777 \\
\hline $0.54-0.78$ & $-3.26 \pm 1.00$ & $0.35 \pm 2.05$ & 0.0985 & 0.129 & 0.0772 \\
\hline $1.26-2.20$ & $-3.50 \pm 1.00$ & $-1.57 \pm 2.03$ & 0.0347 & 0.117 & 0.240 \\
\hline$>2.20$ & $-7.17 \pm 1.10$ & $-6.71 \pm 2.14$ & 0.0047 & 0.0349 & 0.348 \\
\hline
\end{tabular}

Table 2

Measured asymmetries, $A_{\mathrm{fb}}^{\text {raw }}$, and fraction of $Z \rightarrow q \bar{q}$ decays, $\varepsilon_{q}$, in 8 bins of the tagging discriminant for the 1994 and 1995 peak data 


\subsection{Experimental systematic errors}

The detector introduces uncertainties in the measurements both because of possible intrinsic asymmetries and because of the systematic uncertainty on the determination of $P_{\mathrm{S}}$. The following contributions are studied, and the results are summarised in Table 3:

- Angular acceptance: The uncertainty introduced by the detector acceptance is largely reduced by the use of an unbinned maximum likelihood technique. Due to this technique the selection efficiency as a function of $x$ is irrelevant provided it is symmetric in $x$, and thus does not introduce systematic uncertainties. In order to study residual effects at the edges of the tracking devices the measurement is repeated for different values of the acceptance cut.

- Tracking performance and detector material: Asymmetries in the detector response can only bias the measurement if the performance of the tracking is different in the forward and backward regions and for positively and negatively charged tracks. Studies of the tracking system, in particular of the alignment of the ladders of the silicon microvertex detector, show no significant effect.

The quark flavours may be affected by the track selection criteria in different ways, as they fragment with different momentum spectra and different secondary decay characteristics. Therefore the raw asymmetry measurements are repeated varying the selection criteria for tracks used in the jet charge calculation. The following track parameters are studied, and statistically significant varia-

Table 3

Details of the experimental systematic errors on the raw asymmetry $A_{\mathrm{fb}}^{\mathrm{raw}}$

\begin{tabular}{llllll}
\hline Source & \multicolumn{5}{l}{ Contribution to $\Delta A_{\mathrm{fb}}^{\mathrm{raw}}(\%)$} \\
\cline { 2 - 6 } & 1991 & 1992 & 1993 & 1994 & 1995 \\
\hline$P_{\mathrm{S}}$ statistical error & 0.06 & 0.026 & 0.033 & 0.012 & 0.012 \\
$\rho$ uncertainty & 0.008 & 0.009 & 0.008 & 0.007 & 0.004 \\
Acceptance cut & 0.15 & 0.057 & 0.012 & 0.012 & 0.028 \\
Tracking performance & 0.34 & 0.072 & 0.20 & 0.081 & 0.13 \\
Event selection & 0.10 & 0.076 & 0.082 & 0.064 & 0.030 \\
Vertex uncertainties & 0.008 & 0.008 & 0.008 & - & - \\
Total & 0.40 & 0.12 & 0.22 & 0.10 & 0.14 \\
\hline
\end{tabular}

tions in the raw asymmetries are considered as systematic uncertainties:

- The cut on the minimum transverse momentum is varied between $800 \mathrm{MeV}$ and $1.2 \mathrm{GeV}$.

- The cut on the maximum transverse momentum is lowered from 50 to $30 \mathrm{GeV}$.

- The requirements on the minimal track length, on the track quality and the matching to the interaction point are tightened.

Positively and negatively charged particles are known to have different interaction cross sections with the detector materials. To investigate this effect, the measurement is repeated with positively charged tracks only. No significant differences are found.

For 1994 and 1995 data the difference in $b$ tagging efficiency between data and Monte Carlo is taken into account by using the average value. The systematic uncertainty, estimated to be half the difference, has no significant effect.

- Event selection: The effect of background contamination from other processes is negligible. The uncertainties due to the event selection cuts are evaluated by cut variations.

- Vertex position displacements and beam tilts: For the data taken in 1994 and 1995 the directions of calorimetric clusters used to determine the thrust axis refer to the event vertex position as measured using the silicon microvertex detector. For the data taken before 1994 the longitudinal event vertex position was not available, and its nominal position was assumed; the corresponding error on the thrust axis calculation leads to a systematic uncertainty of $0.008 \%$ on the raw asymmetries.

The effects of the slight tilts of the LEP beams with respect to the $z$ axis of the L3 detector were investigated and found to be negligible.

- Errors on correlations: The main source of uncertainty on the calculation of the probability $P_{\mathrm{S}}$ is the error on the correlation between the jet charges in the two hemispheres. The correlation coefficient $\rho$ is extracted from Monte Carlo simulation. The variation of its value with the fragmentation model parameters is taken into account. A relative error of $30 \%$ on the values of $\rho$ is estimated.

The values of $P_{\mathrm{S}}$ are determined from data. Consequently they are known with limited statistical 
Table 4

Effect of the uncertainties on parameters of the hadronisation process on $A_{\mathrm{fb}}^{\mathrm{b}}$ (for $\sqrt{s}=91.24 \mathrm{GeV}$ ) and $\sin ^{2} \bar{\theta}_{\mathrm{W}}$

\begin{tabular}{lll}
\hline Parameter & $\begin{array}{c}\Delta A_{\mathrm{fb}}^{\mathrm{b}} \\
(\%)\end{array}$ & $\begin{array}{l}\Delta \sin ^{2} \bar{\theta}_{\mathrm{W}} \\
\left(\times 10^{4}\right)\end{array}$ \\
\hline$\Lambda_{\mathrm{QCD}}$ & 0.07 & 1.8 \\
$M_{\text {min }}$ & 0.08 & 1.8 \\
Baryon production & 0.17 & 4.0 \\
Fragmentation: general & 0.19 & 4.9 \\
Fragmentation: light flavours & 0.21 & 1.6 \\
$\left\langle x_{E}\right\rangle_{\mathrm{c}}$ & 0.08 & 4.0 \\
$\left\langle x_{E}\right\rangle_{\mathrm{b}}$ & 0.19 & 5.1 \\
B oscillations & 0.12 & 0.1 \\
Total & 0.43 & 9.5 \\
\hline
\end{tabular}

accuracy only. Their statistical errors cause uncertainties on the raw asymmetry measurements, which are also included in Table 3.

\subsection{Theoretical uncertainties}

Theoretical uncertainties enter the determination of $A_{\mathrm{fb}}^{\mathrm{b}}$ and $\sin ^{2} \bar{\theta}_{\mathrm{W}}$ through Eq. (11) by uncertainties on the QCD corrections, $C_{q}$, and on the flavour biases, $\xi_{q}$.

To evaluate the effect due to the uncertainty on the QCD corrections, $C_{q}$, the prescriptions given in Ref. [18] are followed. The full corrections given therein are applied since no requirement on the shape of the event is used. The resulting error on $\sin ^{2} \bar{\theta}_{\mathrm{W}}$ is negligible.

The probability of correct charge assignment of the individual flavours is sensitive to parameters describing the hadronisation process [13]. All of these parameters are tuned on LEP data and are known with limited precision only. They are varied in ranges similar to those in Refs. [19,3,20-23] and the resulting changes in the flavour biases are considered as systematic uncertainties. Parameters influencing the flavour biases are:

- The QCD scale parameter $\Lambda_{\mathrm{QCD}}$ (varied between 240 and $325 \mathrm{MeV}$ );

- The invariant mass cut-off of parton showers $M_{\text {min }}$ (varied between 0.55 and $1.0 \mathrm{GeV}$ );

- Baryon production: the rate of di-quark relative to single quark production $P(q q) / P(q)$ (varied between 0.09 and 0.12), and the parameter in the so-called popcorn model [24] describing how of- ten baryon production is accompanied by the production of high-momentum mesons (varied between 0 and 1 );

- General fragmentation parameters: the ratio of vector / (vector + pseudoscalar) meson production $P_{q}$ (varied between 0.4 and 0.8 for $\mathrm{u}$ and $\mathrm{d}$ quarks, between 0.5 and 0.7 for s quarks, and between 0.65 and 0.85 for $\mathrm{c}$ and $\mathrm{b}$ quarks), the width of the transverse momentum distribution $\sigma_{q}$ (varied between 360 and $420 \mathrm{MeV}$ ), and the rate of SS pairs produced in the fragmentation process relative to $u \bar{u}$ and $\mathrm{d} \overline{\mathrm{d}}$ pairs, $\gamma_{\mathrm{s}}$ (varied between 0.27 and 0.32 );

- The $a$ and $b$ parameters in the Lund symmetric fragmentation function for light flavours (varied between 0.14 and 0.28 , and 0.73 and 0.82 , respectively);

- The average energy fraction carried by hadrons fragmented from heavy flavours, $\left\langle x_{E}\right\rangle_{c}$ and $\left\langle x_{E}\right\rangle_{\mathrm{b}}$ (varied between 0.476 and 0.492 and 0.705 and 0.713 , respectively);

- The B oscillation parameter $x_{\mathrm{d}}$ (varied between 0.6 and 0.7 ).

To evaluate the effect of these uncertainties, a simplified Monte Carlo simulation is used. The flavour biases $\xi_{q}$ are calculated for the values of the parameters listed.

To ensure that the simplified simulation gives reliable results, a reweighting technique is alternatively applied to a sample of fully simulated Monte Carlo events in case of variations of $\Lambda_{\mathrm{QCD}},\left\langle x_{E}\right\rangle_{\mathrm{c}}$ and $\left\langle x_{E}\right\rangle_{\mathrm{b}}$. The uncertainties obtained from the full simulation are in agreement with those derived from the simplified simulation. The resulting uncertainties on the $A_{\mathrm{fb}}^{\mathrm{b}}$ and $\sin ^{2} \bar{\theta}_{\mathrm{W}}$ measurements (see the following Section) are given in Table 4.

Table 5

Errors, $\sigma_{q}$, assigned to the flavour biases $\xi_{q}$ and their correlations, $V_{q q^{\prime}}$

\begin{tabular}{lllllrl}
\hline$q / q^{\prime}$ & $\sigma_{q}$ & $V_{q q^{\prime}}$ & & & & \\
\cline { 3 - 7 } & & $\mathrm{u}$ & $\mathrm{d}$ & $\mathrm{s}$ & $\mathrm{c}$ & $\mathrm{b}$ \\
\hline $\mathrm{u}$ & 0.062 & 1.00 & 0.15 & 0.14 & 0.42 & 0.44 \\
$\mathrm{~d}$ & 0.059 & & 1.00 & 0.49 & 0.15 & 0.25 \\
$\mathrm{~s}$ & 0.046 & & & 1.00 & -0.03 & 0.20 \\
$\mathrm{c}$ & 0.060 & & & & 1.00 & 0.56 \\
$\mathrm{~b}$ & 0.045 & & & & & 1.00 \\
\hline
\end{tabular}


The covariance matrix of the $\xi_{q}$ originating from the parameter uncertainties is derived. The errors and correlations are found to be the same for all data periods. The sum of the individual covariance matrices is listed in Table 5.

\section{Results}

\subsection{Forward-backward b quark asymmetry}

For the data taken in 1994 and 1995, when the silicon microvertex detector was operational, the bquark forward-backward asymmetry, $A_{\mathrm{fb}}^{\mathrm{b}}$, is determined using Eq. (11). To obtain $A_{\mathrm{fb}}^{\mathrm{b}}$ from the raw asymmetry, the contributions from the remaining flavours are subtracted:

$$
A_{\mathrm{fb}}^{\mathrm{b}}=-\frac{A_{\mathrm{fb}}^{\mathrm{raw}}-\sum_{q \neq \mathrm{b}} Q_{q} /\left|Q_{q}\right| f_{q} \xi_{q}\left(1-C_{q}\right) A_{\mathrm{fb}}^{q}}{f_{\mathrm{b}} \xi_{\mathrm{b}}\left(1-C_{\mathrm{b}}\right)} .
$$

The values for $R_{\mathrm{b}}, R_{\mathrm{c}}$, and $A_{\mathrm{fb}}^{\mathrm{c}}$ at $\sqrt{s}=m_{\mathrm{Z}}$ are taken from Ref. [25], and only their small energy dependence is inferred from the Standard Model; the branching ratios and asymmetries for the light flavours are taken from the Standard Model.

The measurement is performed on b-quark enriched sample requiring $D_{\mathrm{tag}}>1.5$, corresponding to a purity $f_{\mathrm{b}}=78 \%$ and an efficiency $\varepsilon_{\mathrm{b}}=55 \%$. Fig. 3(b) shows the b efficiency and purity as a function of the cut value.

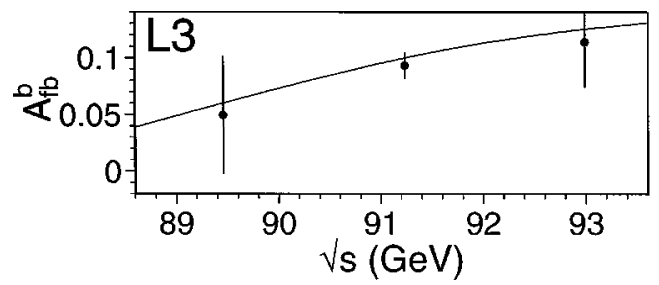

Fig. 4. $A_{\mathrm{fb}}^{\mathrm{b}}$ measurements as a function of centre-of-mass energy. The points represent the measurements made on 1994 and 1995 data; the error bars indicate the statistical uncertainties only. The line represents the Standard Model prediction for $\sin ^{2} \bar{\theta}_{\mathrm{W}}=0.2315$.
Table 6

Input parameters assumed for the measurement of the b-quark forward-backward asymmetry. For each quantity $x$, the partial derivative $\partial A_{\mathrm{fb}}^{\mathrm{b}} / \partial x$ is also given

\begin{tabular}{lcc}
\hline Quantity & Value & Derivative $(\%)$ \\
\hline$A_{\mathrm{c}}$ & 0.0681 & 16.8 \\
$A_{\mathrm{d}, \mathrm{s}}$ & 0.0940 & -4.5 \\
$A_{\mathrm{u}}$ & 0.0602 & 4.7 \\
$R_{\mathrm{b}}$ & 0.2170 & -42.6 \\
$R_{\mathrm{c}}$ & 0.1733 & 6.3 \\
\hline
\end{tabular}

The measured forward-backward asymmetries at the three different center of mass energies are

$A_{\mathrm{fb}}^{\mathrm{b}}=4.95 \pm 5.23 \pm 0.40 \%, \quad \sqrt{s}=89.45 \mathrm{GeV}$,

$A_{\mathrm{fb}}^{\mathrm{b}}=9.31 \pm 1.01 \pm 0.55 \%, \quad \sqrt{s}=91.24 \mathrm{GeV}$,

$A_{\mathrm{fb}}^{\mathrm{b}}=11.37 \pm 3.99 \pm 0.65 \%, \quad \sqrt{s}=92.99 \mathrm{GeV}$.

Fig. 4 compares the measurements to the Standard Model expectations for $\sin ^{2} \bar{\theta}_{\mathrm{W}}=0.2315$ [19]. The measurements are combined, following Ref. [19], to give a determination of the pole asymmetry

$A_{\mathrm{fb}}^{0, \mathrm{~b}}=9.55 \pm 1.07$ (stat.) $\pm 0.55($ syst. $) \%$.

This corresponds to a value of $\sin ^{2} \bar{\theta}_{\mathrm{W}}=0.2329 \pm$ $0.0019 \pm 0.0010$, consistent with the combined value given below.

The correlations with the other forward-backward asymmetries and branching ratios and the central values used for the measurement are listed in Table 6.

\subsection{Effective weak mixing angle}

To determine $\sin ^{2} \bar{\theta}_{\mathrm{W}}$ a $\chi^{2}$ fit to the raw asymmetries is performed using the MINUIT program [26], the $\chi^{2}$ being defined as:

$\chi^{2}=\left(\vec{A}-A^{(\overrightarrow{\mathrm{th}}}\right) \hat{V}^{-1}\left(\vec{A}-A^{\mathrm{th}}\right)$.

where $\vec{A}$ and $A^{\text {th }}$ denote the measured and predicted raw asymmetries, respectively. $A^{\overrightarrow{\mathrm{h}}}$ is obtained using Eq. (11), where the $R_{q}$ and $A_{\mathrm{fb}}^{q}$ are calculated using the ZFITTER program [27]. The covariance matrix $\hat{V}$ 
takes into account the statistical errors and the experimental and theoretical uncertainties with their correlations. The result of the fit for the effective weak mixing angle is

$$
\sin ^{2} \bar{\theta}_{\mathrm{W}}=0.2327 \pm 0.0012(\text { stat. }) \pm 0.0013(\text { syst. }) \text {. }
$$

\section{Acknowledgements}

We wish to express our gratitude to the CERN accelerator divisions for the good performance of the LEP machine. We acknowledge the efforts of all engineers and technicians who have participated in the construction and maintenance of this experiment.

\section{References}

[1] S.L. Glashow, Nucl. Phys. 22 (1961) 579; S. Weinberg, Phys. Rev. Lett. 19 (1967) 1264; A. Salam, Elementary Particle Theory, N. Svartholm (Ed.), Stockholm, Almquist \&Wiksell, 1968, p. 367.

[2] A. Sirlin, Phys. Rev. D 22 (1980) 971; A.A. Akhundov, D. Bardin, T. Riemann, Nucl. Phys. B 276 (1986) 1; D. Bardin et al., Electroweak Working Group Report, CERN Report CERN 95-03, D. Bardin, W. Hollik, G. Passarino (Eds.), and references therein.

[3] ALEPH Collaboration, D. Buskulic et al., Z. Phys. C 71 (1996) 357.

[4] DELPHI Collaboration, P. Abreu et al., Phys. Lett. B 277 (1992) 371.

[5] OPAL Collaboration, P.D. Acton et al., Phys. Lett. B 294 (1992) 436.

[6] R.D. Field, R.P. Feynman, Nucl. Phys. B 136 (1978) 1.

[7] L3 Collaboration, B. Adeva et al., Nucl. Inst. Meth. A 289 (1990) 335 .

[8] M. Acciarri et al., Nucl. Inst. Meth. A 351 (1994) 300.

[9] M. Chemarin et al., Nucl. Inst. Meth. A 349 (1994) 345.

[10] I.C. Brock et al., Nucl. Inst. Meth. A 381 (1996) 236.

[11] A. Adam et al., Nucl. Inst. Meth. A 383 (1996) 342.
[12] L3 Collaboration, M. Acciarri et al., Z. Phys. C 62 (1994) 551.

[13] T. Sjöstrand, PYTHIA 5.7 and JETSET 7.4 Physics and Manual, CERN-TH/7112/93, 1993, revised August 1995; Comp. Phys. Comm. 82 (1994) 74.

[14] KORALZ version 4.01 is used. S. Jadach, B.F.L. Ward, Z. Wa̧s, Comp. Phys. Comm. 79 (1994) 503.

[15] F.A. Berends, P.H. Daverveldt, R. Kleiss, Nucl. Phys. B 253 (1985) 441.

[16] The L3 detector simulation is based on GEANT Version 3.15. R. Brun et al., GEANT 3, CERN-DD/EE/84-1 (Revised), 1987. The GHEISHA program (H. Fesefeldt, RWTH Aachen Report PITHA 85/02, 1985) is used to simulate hadronic interactions.

[17] J. Alcaraz et al., Measurement of the $Z$ Branching Fraction into Bottom Quarks Using Double Tag Methods, L3 Internal Note 2114, 1997.

[18] D. Abbaneo et al., Eur. Phys. J. C 4 (1998) 185.

[19] The LEP Experiments ALEPH, DELPHI, L3 and OPAL, the LEP Electroweak Working Group and the SLD Heavy Flavour Group, A Combination of Preliminary Electroweak Measurements and Constraints on the Standard Model, Preprint CERN-PPE /97-154, 1997.

[20] OPAL Collaboration, R. Akers et al., Z. Phys. C 67 (1995) 365.

[21] L3 Collaboration, M. Acciarri et al., Phys. Lett. B 416 (1998) 220.

[22] The LEP Electroweak Working Group, Presentation of LEP Electroweak Heavy Flavour Results for Summer 1996 Conferences, Internal Note LEPHF /96-01, ALEPH Note 96-099, DELPHI 96-67 PHYS 627, L3 Internal Note 1969, OPAL Technical Note TN391, July 1996.

[23] The LEP B Oscillation Group, Combined Results on $\mathrm{B}^{0}$ Oscillations: Update for the Summer Conferences, Internal Note, LEP-BOSC 97/2, ALEPH 97-083 PHYSIC 97-073 CDF 4297, DELPHI 97-135 PHYS 722, L3 Internal Note 2161, OPAL Technical Note TN502, SLD Physics Note 62, August 1997.

[24] B. Andersson, G. Gustafson, T. Sjöstrand, Phys. Scr. 32 (1985) 574.

[25] Particle Data Group, C. Caso et al., Eur. Phys. J. C 3 (1998) 1.

[26] F. James, CERN Program Library Long Writeup D506 MINUIT, CERN, 1993.

[27] ZFITTER version 5.0 is used. D. Bardin et al., Preprint CERN-TH/6443/92; Z. Phys. C 44 (1989) 493; Nucl. Phys. B 351 (1991) 1; Phys. Lett. B 255 (1991) 290. 